\title{
On the gravitational energy of the Bonnor spacetime
}

\author{
Janusz Garecki \\ Institute of Physics, University of Szczecin, Wielkopolska 15; 70-451 Szczecin, POLAND团
}

(November 13, 2018)

\begin{abstract}
In the paper we consider the gravitational energy and its flux in the Bonnor spacetime. We construct some non-local expressions from the Einstein canonical energy-momentum pseudotensor of the gravitational field which show that the gravitational energy and its flux in this spacetime are different from zero and do not vanish even outside of the material source (a stationary beam of the null dust) of this spacetime.
\end{abstract}

KEY WORDS: gravitational superenergy, gravitational energy-momentum 04.20.Me.04.30.+x

*e-mail:garecki@sus.univ.szczecin.pl 


\section{INTRODUCTION}

In the paper [1] W.B. Bonnor gave the solution to the Einstein equations which describes a stationary beam of light (stricly speaking — a stationary beam of null dust). The line element $d s^{2}$ for this solution reads $(G=c=1)$

$$
d s^{2}=(1+m) d t^{2}-2 m d t d z-(1-m) d z^{2}-d x^{2}-d y^{2}
$$

where

$$
\Delta m:=\frac{\partial^{2} m}{\partial x^{2}}+\frac{\partial^{2} m}{\partial y^{2}}=16 \pi \rho
$$

and $\rho=\mu\left(u^{0}\right)^{2}>0$.

$\mu \geq 0$ is the rest density of the null dust and $:=$ means "by definition".

In the paper [2] were calculated the total energy-momentum "densities", matter and gravity, by using standard energy-momentum complexes of Einstein, Landau-Lifschitz, Papapetrou and Weinberg. All the calculations were performed after transforming the line element (1) to the Kerr-Schild form (see, e.g., [2])

It was shown in [2] that the used four energy-momentum complexes give compatible results in the case and "localize" total energy-momentum "densities" in the Kerr-Schild coordinates to the domains occupied by sources of the gravitational field only, i.e., to the domains in which energy-momentum tensor of the null dust does not vanish. Vacuum regions of the Bonnor spacetime give no energy-momentum contribution, i.e., the gravitational energy-momentum pseudotensors of Einstein, Landau-Lifschitz, Papapetrou and Weinberg globally vanish in the Kerr-Schild coordinates outside of the stationary beam of the considered null dust.

Then, the analogical calculations were repeted in [3] but in Bonnor's coordinates $x^{0}=$

$t, x^{1}=z, x^{2}=x, x^{3}=y$. The results were the same as the results obtained earlier in [2]: in the used coordinates $(t, z, x, y)$ the total energy and momentum "densities" were confined to the regions of non-vanishing energy-momentum tensor of the null dust, i.e., again the vacuum regions of the Bonnor spacetime gave no energy-momentum contribution. 
Despite the fact that their results are coordinate-dependent, the authors of the papers $[2,3]$ conclude that their results sustain the hypothesis which states that the energy and momentum in general relativity (GR) are confined to the regions of non-vanishing energymomentum tensor of matter.1 From this hypothesis it follows, e.g., that the gravitational waves carry no energy-momentum.

In the following we will show that the conclusion of such a kind cannot be correct.

\section{THE GRAVITATIONAL ENERGY AND ITS FLUX IN THE BONNOR SPACETIME OUTSIDE OF THE BEAM}

At first, let us observe that one can write the line element (1)-(2) in the form

$$
d s^{2}=d t^{2}+m(d t-d z)^{2}-d z^{2}-d x^{2}-d y^{2},
$$

where

$$
\Delta m=16 \pi \rho \geq 0 .
$$

Then, after introducing new variables $u=t-z, v=t+z$ we get

$$
\begin{gathered}
d s^{2}=m d u^{2}+d u d v-d x^{2}-d y^{2}, \\
\Delta m=16 \pi \rho \geq 0 .
\end{gathered}
$$

Finally, by introducing $U=\frac{u}{\sqrt{2}}, V=\frac{v}{\sqrt{2}}, X=x, Y=y$ one gets the line element (1)-(2) in the form

$$
d s^{2}=2 m d U^{2}+2 d U d V-d X^{2}-d Y^{2},
$$

where

\footnotetext{
${ }^{1}$ This old hypothesis was recently restored, e.g., by F. Cooperstock
} 


$$
\Delta m=\frac{\partial^{2} m}{\partial X^{2}}+\frac{\partial^{2} m}{\partial Y^{2}}=16 \pi \rho .
$$

In vacuum, i.e., outside of the stationary beam of the null dust which is the source of the Bonnor spacetime, we obtain from (7)-(8)

$$
\begin{gathered}
d s^{2}=2 m(X, Y) d U^{2}+2 d U d V-d X^{2}-d Y^{2}, \\
\Delta m=\frac{\partial^{2} m}{\partial X^{2}}+\frac{\partial^{2} m}{\partial Y^{2}}=0,
\end{gathered}
$$

i.e., we obtain special plane-fronted gravitational wave with parallel rays (p-p wave, see, e.g., $[4])$.

In an old our paper [5] it was shown that in the coordinates $(U, V, X, Y)$ the canonical Einstein's gravitational energy-momentum pseudotensor ${ }_{E} t_{i}{ }^{k}$ globally vanishes for the pp waves giving zero gravitational "energy density" and no gravitational energy flux, in agreement with the results of the papers $[2,3]$. But if one transforms the line element (9)(10) to the coordinates used by MTW [6], then one will obtain non-vanishing pseudotensor ${ }_{E} t_{i}{ }^{k}$ and nonzero (negative) "energy density" and its non-zero flux (see, e.g., [5]).

So, as it is commonly known, the results obtained by using of the energy-momentum pseudotensors of the gravitational field (and complexes) are coordinate - dependent and one must not use them for supporting the hypothesis about "localization of the gravitational energy to the regions of the non-vanishing energy-momentum tensor of the matter and all non-gravitational fields".

The energy-momentum complexes, matter and gravitation, and in consequence the gravitational energy-momentum pseudotensors, can only be reasonably use in a case of very precisely defined asymptotically flat, in null or in spatial infinities, spacetime giving global (or integral) energy and momentum. But they cannot have physical meaning to any local analysis of the gravitational field.

In order to do a reasonable and covariant local analysis of the gravitational field one should use covariant expressions constructed from the curvature tensor, e.g., one should use 
the canonical superenergy tensor ${ }_{g} S_{i}{ }^{k}\left(P ; v^{l}\right)$ of the gravitational field (and canonical angular supermomentum tensor).

The tensor of such a kind was introduced in series of the our papers [7-12]. In vacuum, the canonical superenergy tensor of the gravitational field has the following form

$$
\begin{aligned}
{ }_{g} S_{i}{ }^{k}\left(P ; v^{l}\right) & =\frac{8 \alpha}{9}\left(2 v^{a} v^{b}-g^{a b}\right)\left[R_{(a \mid}^{k l m} R_{i(l m) \mid b)}\right. \\
& \left.-\frac{1}{2} \delta_{i}^{k} R_{(a \mid}^{l m n} R_{l(m n) \mid b)}\right]
\end{aligned}
$$

where $\alpha=\frac{1}{16 \pi}$ and Latin indices run over the values $0,1,2,3$.

Here $v^{a} v_{a}=1$ means a 4 -velocity of an observer $\mathbf{O}$ which is studying gravitational field and round brackets denote symmetrization. The indices inside vertical lines, e.g., $(a|b c| d)$ are excluded from symmetrization.

The tensor (11) was obtained as a result of a special averaging of the differences ${ }_{E} t_{i}{ }^{k}(y)-$ ${ }_{E} t_{i}{ }^{k}(P)$ in a Riemann normal coordinates $\mathbf{N C}(\mathbf{P})$ introduced in a sufficiently small vicinity of an (arbitrary) point $\mathbf{P}$ (For details-see, e.g., [7-12]). So, it is some kind of a non-local construction obtained from the Einstein canonical energy-momentum pseudotensor ${ }_{E} t_{i}{ }^{k}$.

The fiducial observer $\mathbf{O}$ is at rest in this $\mathbf{N C}(\mathbf{P})$. In Bonnor's coordinates $(t, z, x, y)$ the four-velocity $\vec{v}$ of this observer has the following components

$$
v^{i}=\frac{\delta_{0}^{i}}{\sqrt{g_{00}}}=\frac{\delta_{0}^{i}}{\sqrt{1+m}}, \Rightarrow v_{i}=\frac{g_{i 0}}{\sqrt{1+m}} .
$$

In the paper [5] were calculated the components ${ }_{g} S_{i}{ }^{k}\left(P ; v^{l}\right)$ of the canonical superenergy tensor (11) for a gravitational p-p wave in the null coreper

$$
\vartheta^{0}=m d U+d V, \vartheta^{1}=d U, \vartheta^{2}=d X, \vartheta^{3}=d Y
$$

determined by the line element (9)-(10). There was obtained that the only one component

$$
{ }_{g} S_{0}{ }^{1}=\frac{16 \alpha}{9}\left(v^{1}\right)^{2}\left[\left(m_{x x}\right)^{2}+2\left(m_{x y}\right)^{2}+\left(m_{y y}\right)^{2}\right]
$$

\footnotetext{
${ }^{2}$ At every point $\mathbf{P}$ we have chosen the unit timelike vector of the $\mathbf{N C}(\mathbf{P})$ to be proportional to the timelike vector of the holonomic frame at the point determined by the Bonnor's coordinates.
} 
of the ${ }_{g} S_{i}{ }^{k}\left(P ; v^{l}\right)$ is different from zero in the coreper (13).

Here and in the following $m_{x x}=\frac{\partial^{2} m}{\partial x^{2}}, m_{x y}=\frac{\partial^{2} m}{\partial x \partial y}, m_{y y}=\frac{\partial^{2} m}{\partial y^{2}}$.

By using (14), one can easily calculate the canonical superenergy density ${ }^{g} \epsilon_{s}$ of the p-p wave (9)-(10) in the null coreper

$$
\begin{aligned}
{ }^{g} \epsilon_{s} & :={ }_{g} S_{i}{ }^{k} v^{i} v_{k} \doteq{ }_{g} S_{1}{ }^{0}\left(v^{1}\right)^{2} \doteq{ }_{g} S_{1}{ }^{0}\left(v_{0}\right)^{2} \\
& =\frac{4 \alpha}{9(m+1)^{2}}\left[\left(m_{x x}\right)^{2}+2\left(m_{x y}\right)^{2}+\left(m_{y y}\right)^{2}\right]>0 .
\end{aligned}
$$

The sign $\doteq$ means that an equation is valid only in some special coordinates or frames.

The expression (15), like the expression (14), is positive-definite and it does not vanish in any coordinates or frames.

Also the spatial Poynting's supervector

$$
{ }_{g} P^{i}:=\left(\delta_{k}^{i}-v^{i} v_{k}\right)_{g} S_{l}{ }^{k} v^{l}
$$

does not vanish in the case and it has the two non-vanishing components $P^{0}, P^{1}$ in the null coreper (13):

$$
\begin{gathered}
P^{0} \doteq \frac{8 \alpha}{9 \sqrt{2(m+1)^{3}}}\left[\left(m_{x x}\right)^{2}+2\left(m_{x y}\right)^{2}+\left(m_{y y}\right)^{2}\right], \\
P^{1} \doteq \frac{-8 \alpha}{9(m+1) \sqrt{2(m+1)^{3}}}\left[\left(m_{x x}\right)^{2}+2\left(m_{x y}\right)^{2}+\left(m_{y y}\right)^{2}\right]=\frac{-P^{0}}{(m+1)} .
\end{gathered}
$$

So, we have for Bonnor spacetime (1)-(2) the non-vanishing gravitational superenergy density and non-vanishing gravitational superenergy flux even outside of the sources of this spacetime, i.e., outside of the domains in which $T_{i}^{k} \neq 0$.

As we have used tensorial expressions only in our analysis of the Bonnor spacetime, the our results are valid in any coordinates or frames. Thus, one can conclude that in the case of the Bonnor spacetime one has a positive-definite gravitational superenergy density and non-vanishing gravitational superenergy flux in vacuum, i.e., outside of the stationary beam 
of the null dust which is the source of this spacetime.

But this means that the free gravitational field in the Bonnor spacetime for which $R_{i k l m} \neq$ 0 also possesses its own gravitational (relative) energy-density and the non-zero of this (relative) gravitational energy flux. It is easily seen from the following considerations.

Let us consider an observer $\mathbf{O}$ which is studying gravitational field. His world-line is $x^{a}=x^{a}(s)$, and $\vec{v}: v^{a}=\frac{d x^{a}}{d s}$ represents his four-velocity. At any point $\mathbf{P}$ of the world-line one can define an instantaneous, local 3-space of the observer $\mathbf{O}$ orthogonal to $\vec{v}$. This instantaneous 3-space has the following interior proper Riemannian metric

$$
\gamma_{a b}=v_{a} v_{b}-g_{a b} \doteq\left(\frac{g_{0 a} g_{0 b}}{g_{00}}-g_{a b}\right) \doteq \gamma_{\alpha \beta} \doteq(-) g_{\alpha \beta},
$$

where the Greek indices run over the values 1,2,3 (see, e.g., [13]).

Then, by using the gravitational superenergy density ${ }^{g} \epsilon_{s}$ and its flux ${ }_{g} P^{i}$, one can easily construct in such instantaneous local 3-space of the observer O, e.g., the following non-local expressions which have proper dimensions of the energy density and its flux

$$
\begin{aligned}
\epsilon_{e n} & :=\oint_{S_{2}}{ }^{g} \epsilon_{s}(P) d^{2} S \\
& \approx{ }^{g} \epsilon_{s}(P) \oint_{S_{2}} d^{2} S, \\
P^{i} & :=\oint_{S_{2}} P^{i}(P) d^{2} S \\
& \approx{ }_{g} P^{i}(P) \oint_{S_{2}} d^{2} S .
\end{aligned}
$$

\footnotetext{
${ }^{3}$ Of course, the same conclusion is also true inside of the beam. However, in this domain the suitable expression on ${ }_{g} S_{i}{ }^{k}\left(P ; v^{l}\right)$ is slightly modified by additional terms which depend on the matter tensor $T_{i}^{k}$. On the other hand, the canonical superenergy tensor of matter ${ }_{m} S_{i}{ }^{k}\left(P ; v^{l}\right)$ defined in [7-12] is confined to the domains occupied by matter, i.e., it is confined to the beam only.
} 
Here $S_{2}$ means a small sphere $\gamma_{\alpha \beta} x^{\alpha} x^{\beta}=R^{2}$ in the instantaneous local 3-space of the observer $\mathbf{O}$.

$$
d^{2} S=\sqrt{\gamma_{\alpha \beta} \sigma^{\alpha} \sigma^{\beta}}
$$

where

$$
\sigma^{\alpha}=\frac{1}{2} \gamma^{\alpha \beta} \epsilon_{\beta \gamma \delta} d x^{\gamma} \wedge d x^{\delta}
$$

and $\gamma^{\alpha \beta}$ means the inverse metric to the interior metric $\gamma_{\alpha \beta}$.

$\epsilon_{\alpha \beta \gamma}$ is the 3-dimensional Levi-Civita pseudotensor established by the condition

$$
\epsilon_{123}=\sqrt{\gamma}
$$

where $\gamma:=\operatorname{det}\left(\gamma_{\alpha \beta}\right)$. The expressions (20)-(21) give us the relative gravitational energy density and its flux for an observer $\mathbf{O}$ in his instantaneous 3-space orthogonal to $\vec{v}$. values of these quantities $\epsilon_{e n}$ and $P^{i}$ depend on radius $R$ of the sphere $S_{2}$ also. In order to get unique $\epsilon_{e n}$ and $P^{i}$ one can take as the sphere $S_{2}$, e.g., the smallest classical sphere admitted by the loops quantum gravity.

This quantum theory of gravity tells us (see, e.g., [14-23]) that one can say about continuous classical differential geometry already just a few orders of magnitude above the Planck scale, e.g., for distances $L \approx 100 L_{P} \approx 10^{-33} \mathrm{~m}$. So, one can take the radius $R$ of the smallest classical sphere in an instant 3-space of the observer $\mathbf{O}$, orthogonal to $\vec{v}$, to be of order $R \approx 100 L_{P}=100 \cdot \sqrt{\frac{G \hbar}{c^{3}}} \approx 10^{-33} \mathrm{~m}$. In such a case, when calculating the integrals (20)-(21) one can assume that their integrands are constant during integration and evaluate these integrals in the way as it was already done in (20)-(21).

As we could have seen from this paper the two quantities $\epsilon_{e n}$ and $P^{i}$ do not vanish in the Bonnor spacetime for every $\mathbf{O}$; especially, they do not vanish outside of the beam which is

\footnotetext{
${ }^{4}$ The fact that (20)-(21) give us some kind of the relative quantities (with respect $\mathbf{P}$ ) is seen from the method of the construction of the canonical superenergy tensors (see, e.g., [7-12]).
} 
the source of this spacetime. In consequence, one can conclude that it is easy to attribute to the gravitational field in Bonnor spacetime the positive-definite (although relative) energydensity and its non-zero flux by using, e.g., our canonical superenergy tensor of this field.

When we only use gravitational pseudotensors, then this important fact is camouflaged in some coordinates, like Kerr-Schild coordinates or Bonnor coordinates, by energy and momentum of the inertial forces field (with $R_{i k l m}=0$ ) which simply cancels (outside of the beam) with energy and momentum of the real gravitational field (with $R_{i k l m} \neq 0$ ).

Thus, the conclusions of the authors of the papers $[2,3]$ are incorrect as they resulted from coordinate-dependent pseudotensorial expressions. In order to get the correct information about gravitational energy and momentum (and also about gravitational angular momentum, see, e.g., $[11,12])$ one must use covariant expressions which depend on the curvature tensor, like our canonical gravitational superenergy tensor ${ }_{g} S_{i}{ }^{k}\left(P ; v^{l}\right)$ (and like our gravitational angular supermomentum tensors, see, e.g., $[11,12])$. 


\section{REFERENCES}

[1] W.B. Bonnor, Gen.Rel.Grav., 32 (2000) 1627.

[2] T. Bringley, "Energy and momentum of a stationary beam of light", arXiv:gr$\mathrm{qc} / 0204006$.

[3] R.M. Gad, "Energy distribution of a stationary beam of light", arXiv:gr-qc/0307010.

[4] J. Ehlers and W. Kundt, an article in "Gravitation: an Introduction to Current Problems", ed. L. Witten, John Wiley, New York 1962.

[5] J. Garecki, Rep.Math. Phys., (1997) 485.

[6] C.W. Misner, K.S. Thorne and J.A. Wheeler, "Gravitation", W.H. Freeman and Company, San Francisco 1973.

[7] J. Garecki, Rep.Math.Phys., 33 (1993) 57.

[8] J. Garecki, J.Math.Phys., 40 (1999) 4035.

[9] J. Garecki, Rep.Math.Phys., 43 (1999) 397.

[10] J. Garecki, Rep.Math.Phys., 44 (1999) 95.

[11] J. Garecki, Ann.Phys.(Leipzig), 11 (2002) 441.

[12] M.P. Dạbrowski and J. Garecki, Class. Quantum Grav., 19 (2002) 1.

[13] L.D. Landau and E.M. Lifschitz, "The Classical Theory of Fields", Pergamon, Oxford 1975.

[14] A. Ashtekar, "Quantum Mechanics of Geometry", arXiv:gr-qc/9901023.

[15] A. Ashtekar, "Quantum Geometry and Gravity: Recent Advances", arXiv:grqc/0112038.

[16] J. Pullin, "Canonical quantization of general relativity: the last 18 years in a nutshell", arXiv:gr-qc/0209008.

[17] C. Rovelli, "A dialog on quantum gravity", arXiv:hep-th/0310077. 
[18] F. Markopoulou, "Planck-scale models of the Universe", arXiv:gr-qc/0210086.

[19] A. Ashtekar, "Quantum Geometry In Action: Big-Bang and Black Holes", arXiv:math$\mathrm{ph} / 0202008$.

[20] M. Bojowald, "Loop Quantum Cosmology: Recent Progress", arXiv:gr-qc/0402053.

[21] A. Ashtekar and J. Lewandowski, "Background Independent Quantum Gravity: A Status Report", arXiv:gr-qc/0404018.

[22] L. Smolin, "How far we are from the quantum theory of gravity"?, arXiv:hepth/0303185.

[23] A. Ashtekar, "Gravity and the Quantum", arXiv:gr-qc/0410054. 